\title{
PROBABLE SPIN-ORBIT ALIGNED SUPER-EARTH PLANET CANDIDATE KOI2138
}

\author{
Jason W. Barnes ${ }^{1,2}$, Johnathon P. Ahlers ${ }^{1}$, Shayne A. Seubert ${ }^{1}$, and Howard M. Relles ${ }^{3}$ \\ ${ }^{1}$ Department of Physics, University of Idaho, Moscow, ID 83844-0903, USA; jwbarnes@uidaho.edu \\ Received 2015 March 20; accepted 2015 July 2; published 2015 July 27
}

\begin{abstract}
We use rotational gravity darkening in the disk of Kepler star KOI-2138 to show that the orbit of $2.1-R_{\oplus}$ transiting planet candidate KOI-2138.01 has a low projected spin-orbit alignment of $\lambda=1^{\circ} \pm 13^{\circ}$. KOI-2138.01 is just the second super-Earth with a measured spin-orbit alignment after 55 Cancri e, and the first to be aligned. With a 23.55 days orbital period, KOI-2138.01 may represent the tip of a future iceberg of solar-system-like terrestrial planets having intermediate periods and low-inclination circular orbits.
\end{abstract}

Key words: planets and satellites: individual (KOI-2138)

\section{INTRODUCTION}

Radial velocity and transit surveys have discovered a dramatic variety of planetary system architectures. Evidently, the planet formation process need not necessarily proceed as it has in the solar system. All of our solar system planets orbits within $7^{\circ}$ of the Sun's equatorial plane (Lissauer 1993), an angle that we will call the planets' spin-orbit alignment angle, $\varphi$. The Sun's planets' spin-orbit alignment indicates that they formed from a disk without significant subsequent changes in orbital inclination.

Similarly, measurement of exoplanet spin-orbit alignments can probe those planets' formation and subsequent orbital evolution. Determination of the spin-orbit alignment $\varphi$ for Hot Jupiters has provided primary evidence for ascertaining their origins. Many investigations (e.g., Winn et al. 2010; Johnson et al. 2011; Bieryla et al. 2015) used ground-based radial velocity observations of the Rossiter-McLaughlin effect (McLaughlin 1924; Rossiter 1924; which is sensitive to a planet's projected alignment but not to the orientation of the stellar spin axis with respect to the plane of the sky) to show that Hot Jupiters around more massive stars are more likely to be spin-orbit misaligned than are planets around lower-mass stars. This difference in alignments probably owes to evolution, not origins.

Albrecht et al. (2012) showed that the transition between mostly aligned systems and mostly misaligned systems occurs near a stellar effective temperature of $T_{\text {eff }} \sim 6200 \mathrm{~K}$. Because this is the border between convective and radiative envelopes for stars, Albrecht et al. (2012) postulated that alignment for lower-mass stars results from tidal interactions. Later-type, convective stars have higher tidal dissipation and thus lower tidal quality factors $(Q)$ than earlier-type, radiative stars. The result is that tides induced on the star by the planet exchange angular momentum more effectively for low-mass stars.

Ultimately, this result suggests that Hot Jupiters around lowmass stars are spin-orbit aligned because the planets pull the stellar spin into alignment over time via tides-not because these systems were formed in an aligned state. The recent discovery of a highly spin-orbit misaligned planet around brand-new low-mass pre-main-sequence star PTFO 8-8695 (van Eyken et al. 2012; Barnes et al. 2013) corroborates the

\footnotetext{
2 ResearcherID: B-1284-2009.

3 http://exoplanet-science.com/
}

story that Hot Jupiters acquire orbits isotropically distributed in space ("random" alignments) early in their history.

More recent work has used the distribution of measured spin-orbit alignments to evaluate possible mechanisms to generate spin-orbit misaligned planets in the first place (see Crida \& Batygin 2014 and references therein). Initial results indicate that single proposed mechanisms have difficulty reproducing the observed distribution. Different systems may therefore produce misalignment in different ways. Production mechanisms for the misalignment for smaller, non-giant worlds have not seen extensive consideration.

Because the Rossiter-McLaughlin effect's signal goes as $\frac{R_{p}^{2}}{R_{*}^{2}}$ (where $R_{p}$ and $R_{*}$ are the planetary and stellar radius, respectively), observations to characterize the origins and evolution of those smaller, non-giant planets becomes progessively more difficult with decreasing planet radius. Some researchers (Hirano et al. 2012b; Morton \& Winn 2014) have worked around this challenge by constraining the stellar axis tilt with respect to the plane of the sky (the stellar obliquity $\psi$ ) directly by comparing the stellar radius, rotation period, and projected rotational velocity. An obliquity measurement alone constrains but does not directly measure transiting planets' spin-orbit alignments. While a non-zero stellar obliquity $\psi$ requires that any transiting planets be misaligned, a measured obliquity of zero allows but does not require spin-orbit alignment. Morton \& Winn (2014) thereby indirectly confirmed spin-orbit misalignment for several super-Earths and super-Earth candidates: Kepler-96b, KOI269.01, KOI323.01, KOI355.01, KOI974.01, KOI1890.01, KOI2002.01, KOI2026.01, and KOI2261.01. Similarly, asteroseismological determination of a nonzero stellar obliquity $\psi$ by Chaplin et al. (2013) showed that two super-Earths orbiting Kepler-50 and three super-Earths around Kepler-65 must be misaligned.

Multiple-planet systems can indirectly confirm aligned super-Earths. If more than one planet around a given star transits, then the likelihood of planet coplanarity increases dramatically (e.g., Lissauer et al. 2011). Therefore, RossiterMcLaughlin measurements of a giant planet can imply a similar alignment for any other planets in that same system that may be too small to measure directly. Hence the Rossiter-McLaughlin determination of spin-orbit alignment by Hirano et al. (2012a) and Albrecht et al. (2013) for the giant planet (KOI-94.01) in the KOI-94 system also implies a probable alignment for the 3.73 day period super-Earth in that system (KOI-94.04). 
The only super-Earth with a directly measured projected spin-orbit alignment to date is 55 Cancri e $(55 \mathrm{Cnc}$ e; Bourrier $\&$ Hébrard 2014). The fourth planet detected of five now known in the 55Cnc system (Fischer et al. 2008), 55Cnc e has an orbital period of just 0.7365 days (Dawson \& Fabrycky 2010). Its Rossiter-McLaughlin-measured projected misalignment of $72^{\circ} \pm 12^{\circ}$ indicates an askew orbit relative to both the stellar equator and its sibling planets (McArthur et al. 2004). The 55Cnc system has clearly experienced a very different history than that of our own solar system.

The origin and evolution of longer-period (not tidally influenced) planets are not yet constrained by spin-orbit measurements. The Rossiter-McLaughlin effect requires a complete transit to be visible in a given night of ground-based observing. Thus Rossiter-McLaughlin measurements for longer period planets are more difficult because (1) they transit less frequently and (2) their transits have longer duration. Additional techniques have successfully measured spin-orbit alignments of giant planets as well, particularly Doppler tomography (Collier Cameron et al. 2010a, 2010b; Miller et al. 2010; Gandolfi et al. 2012; Johnson et al. 2014), stroboscopic starspots (Désert et al. 2011; Nutzman et al. 2011; Sanchis-Ojeda et al. 2011; Sanchis-Ojeda \& Winn 2011; Tregloan-Reed et al. 2013), and asteroseismology (Chaplin et al. 2013; Huber et al. 2013).

Herein we use another technique that relies on rapid stellar rotation: gravity darkening. Stellar rotation causes a lower effective surface gravity $(g)$ at the equator than at the pole due to centrifugal force. Lower surface gravity leads to a larger scale height in the stellar atmosphere, which von Zeipel (1924) showed leads to cooler photospheric temperatures. Those lower temperatures lead to lower emitted flux from the equator than from the pole, which we call gravity darkening. The Von Zeipel Theorem shows that the emitted flux from a gravitydarkened stellar photosphere is proportional to the local surface gravity. Fast-rotating stars therefore have hotter and brighter poles and cooler and dimmer equators. Gravity darkening has now been directly observed by optical interferometric observations of Vega ( $\alpha$ Lyrae; Peterson et al. 2006, explaining residuals in earlier near-IR interferometry by Ciardi et al. 2001) and Altair ( $\alpha$ Aquilae; Monnier et al. 2007). Eclipsing binary stars have long been analyzed using lightcurves across gravitydarkened stellar disks (i.e., Djurašević et al. 2003).

Barnes (2009) showed that the spin-orbit alignment for planets orbiting rapidly rotating stars can be determined from transit photometry alone by taking advantage of gravity darkening. The gravity darkening-induced nonuniformity of the stellar disk introduces characteristic asymmetries into misaligned planets' transit lightcurves. Careful fitting of the precise lightcurve can then constrain an orbiting planet's spinorbit alignment. Because it requires photometry from only a single transit, gravity darkening can be applied to planets of any orbital period and thus works well for long-period Kepler transits with existing lightcurves.

Gravity darkening has already been leveraged to measure the spin-orbit misalignment in four systems. Szabó et al. (2011) first found an asymmetry in the Kepler transit lightcurve of KOI-13 that they attributed to spin-orbit misalignment around a fast-rotating star-consistent with the Barnes (2009) predictions. Barnes et al. (2011) then fit KOI-13.01's asymmetric transit lightcurve with a gravity-darkened stellar transit model to test whether or not gravity darkening could explain the measured signature. It can. KOI-13.01 has a spinorbit misalignment of $56^{\circ} \pm 4^{\circ}$ (Barnes et al. 2011). The lightcurve analysis in Barnes et al. (2011) is degenerate, so a retrograde spin-orbit alignment of $126^{\circ} \pm 4^{\circ}$ is also possible.

Gravity darkening can also indicate spin-orbit alignment in symmetric or nearly symmetric lightcurves like that of KOI368 (Zhou \& Huang 2013; Ahlers et al. 2014). In this case, it is the lack of asymmetry in the lightcurve of a planet around a star with sufficiently high $v \sin \psi$ (where $\psi$ is the stellar obliquity relative to the plane of the sky, equivalent to the traditional stellar $v \sin i$ ) that constrains alignment (but see also Zhou \& Huang 2013). More recently, gravity darkened lightcurves revealed nodal precession for exoplanets KOI-13 (Masuda 2015) and PTFO 8-8695 (van Eyken et al. 2012; Barnes et al. 2013) and mutual alignment for two transiting planetary candidates (KOI-89.01 and KOI-89.02) that are misaligned with their parent star (J. P. Ahlers et al. 2015, in preparation).

In this letter we analyze the Kepler transit lightcurve of planet candidate KOI-2138.01 to measure its spin-orbit alignment. At 2.1 Earth radii, KOI-2138.01 is only the second super-Earth candidate for which spin-orbit alignment has been measured, after 55 Cancri e. In Section 2 we describe the system's parent star and the Kepler photometry from which we generate a transit lightcurve. We fit that lightcurve in Section 3 before wrapping up with a discussion of the implications of our measurement in Section 4.

\section{OBSERVATIONS}

The threshold-crossing-event that gave rise to Kepler Object of Interest (KOI) 2138 was first discovered by Batalha et al. (2013). The most recent Kepler parameters for the parent star show that it is an early-type $2.335 M_{\odot}$ star with $T_{\text {eff }}=9565 \mathrm{~K}$ (Rowe et al. 2015). The transit depth of the planet is only $86 \mathrm{ppm}\left(8.6 \times 10^{-5}\right)$, so although the star has a relatively bright Kepler magnitude of $m_{\mathrm{Kep}}=11.98$, the signal-to-noise ratio $(\mathrm{S} / \mathrm{N})$ of the folded transit is just $\mathrm{S} / \mathrm{N}=30$ (Rowe et al. 2015).

The star's rapid rotation piqued our interest, as fast stellar rotation drives the gravity darkening effect. The faster a star rotates, the more gravity darkened it gets. On the Kepler Community Follow-up Observation Program site, Allyson Bieryla and colleagues report a spectroscopic $v \cos \psi$ for KOI2138 of $200 \mathrm{~km} \mathrm{~s}^{-1}$ _fast enough that the star should be severely gravity-darkened. In such high gravity darkening cases the total deviation in transit depth from a non-rotating star case can be up to a factor of 2 (Barnes 2009), and thus eminently visible even in a low signal-to-noise transit such as this one.

We show the Kepler long-cadence photometry of the KOI2138.01 transit in Figure 1. No KOI-2138 data exist for Kepler Quarters 6, 10, and 14 due to failure of one of the photometer's detectors. We use pre-search data conditioned (PDC) fluxes for our analysis - the KOI-2138.01 transit is subtle enough to be very difficult to identify in raw photometry. To eliminate systematic stellar and instrumental variations in the lightcurve, we median boxcar filter the data with a period of $44 \mathrm{hr}(\sim 6$ times the transit duration of $7.1 \mathrm{hr}$ ) to remove stellar and instrumental trends. As this filter has not previously been used on such a low signal-to-noise transit, we analyzed its efficacy by generating simulated lightcurves with similar photon shot noise $\left(6 \times 10^{-5}\right)$ and Gaussian-distributed random linear trends 


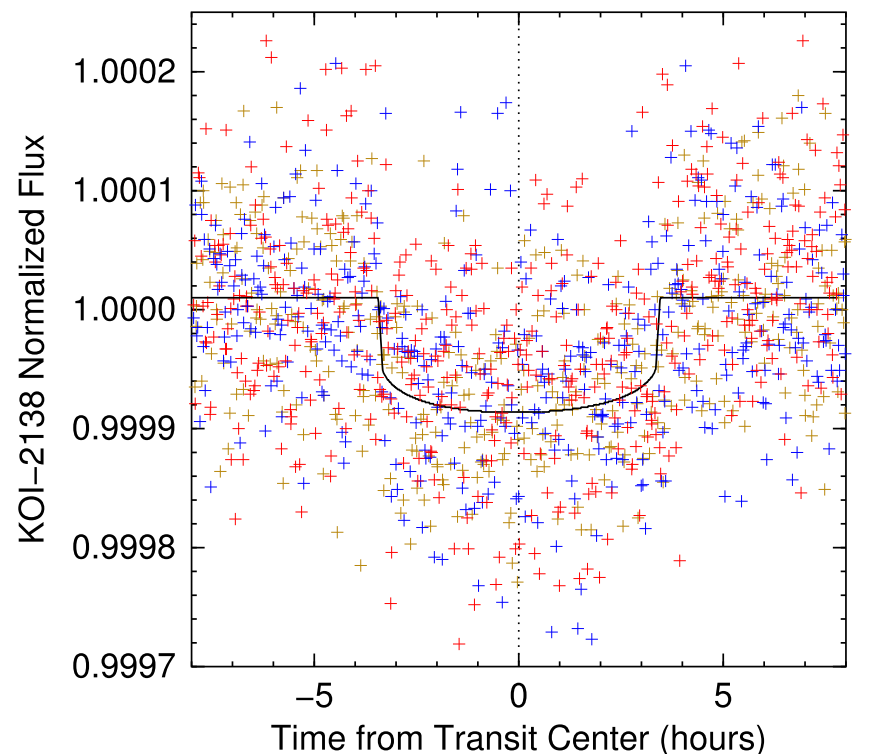

Figure 1. Processed Kepler data before binning and as a function of observation Quarter. Quarter 1 (i.e., all quarters for which the Quarter number $Q \bmod 4=1$ ) is brown, Quarter 3 is red, and Quarter 4 is blue. Quarter 2 is not shown because data from Quarters 6,10, and 14 were lost due to a CCD failure on board the spacecraft. The black line indicates best-fit transit lightcurve.

with total differences of $10^{-4}$ over the $44 \mathrm{hr}$ filter period. When we process these simulated data in the same way as we do the Kepler PDC data, the resulting processed lightcurves retained any original symmetry or asymmetry of a small transit of the same depth as that of KOI-2138.01. The median boxcar cannot account for higher-order variability on timescales shorter than the boxcar duration, however, and we rely on phase-folding of the data to average out any variations on those shorter timescales.

No trends or variations are evident when the timeseries are analyzed as a function of Kepler viewing geometry (i.e., looking at Quarters 1, 5, 9, 13, and 17 together because all were acquired with the same spacecraft orientation and similar for the Quarter 2, 3, and 4 geometry; see Figure 1). The KOI2138 transit lightcurve shows no evidence for significant transit timing variations, either-the strongest periodicity in a LombScargle periodogram of the $O-C$ times occurs with a false alarm probability of 0.269 at 49.93 days. Therefore we folded the 44 transits with data at the 23.5540725 day MAST-listed period. Because experience indicates that only systems with false alarm probabilities less than 0.01 possess credible transit timing variations, our folding the lightcurve at the observed period does not adversely affect the resulting analysis. And folding the data improves the signal-to-noise of the final lightcurve while averaging out variable systematic influences as may arise from PDC conditioning, the median boxcar filter step, or inherent stellar variability.

\section{CONSTRAINTS}

To constrain the spin-orbit alignment $\varphi$ of planet candidate KOI-2138.01, we fit the lightcurve using the Barnes (2009) transitfitter algorithm. This program numerically integrates flux from gravity-darkened stars and fits the resulting lightcurves using a Leavenberg-Marquardt approach from Press et al. (2007) with time-integration. Although the S/N of the transit is low (29.9; Rowe et al. 2015), the large deviations from symmetry expected for a misaligned system (e.g., the red and green curves in Figure 2) allow us to rule out some projected alignments $\lambda$.

The small planet radius leads to rapid transit ingress and egress, unresolved by Kepler long-cadence photometry. Hence in our fits we fix the stellar radius at the MAST value of $2.286 R_{\odot}$. The low signal-to-noise cannot independently constrain the stellar limb darkening; therefore we fix the $c_{1}$ $\left(=u_{1}+u_{2}\right)$ limb darkening coefficient at the value measured for KOI-13 (Barnes et al. 2011), which is at a similar $T_{\text {eff }}$ (but see also Masuda 2015). We hold the stellar $v \cos \psi$ fixed at $200 \mathrm{~km} \mathrm{~s}^{-1}$, which sets the intensity of stellar gravity darkening. (We also assume that the gravity darkening parameter $\beta=0.25$ as expected for stars with radiative envelopes; von Zeipel 1924.) However, the $v \cos \psi=200 \mathrm{~km} \mathrm{~s}^{-1}$ measurement is a lower limit to the star's rotational velocity because it represents the highest valid $v \cos \psi$ from the template spectra (D. Latham 2015, personal communication); thus more stringent constraints may be possible with better stellar $v \cos \psi$ determination.

We show the best-fit lightcurve in Figure 2, and the best-fit parameters in Table 1 . We depict the transit model graphically in Figure 3. The stellar obliquity $\psi$, defined as how far the star's north pole is tilted away from the plane of the sky, is only very poorly constrained $\left(\psi=-4^{\circ} \pm 60^{\circ}\right)$. In fact a firstprinciples calculation shows a similar constraint: if $\psi$ were beyond $\pm 60^{\circ}$ the star would be rotating beyond its breakup speed with $v \cos \psi=200 \mathrm{~km} \mathrm{~s}^{-1}$. Without a more robust estimation of the stellar obliquity the true spin-orbit alignment of this system will remain unknown.

We derive more useful constraints on the projected alignment $\lambda$, defined as the direction of the planet's velocity vector at inferior conjunction measured clockwise from the $x$ axis (to the right in Figure 3 ). The formal uncertainty from the fit covariance matrix yields $\lambda=1^{\circ} \pm 13^{\circ}$ - consistent with KOI-2138.01 in spin-orbit alignment.

A more thorough error analysis shows, however, that valid models with $\lambda$ up to $60^{\circ}$ or as low as $-20^{\circ}$ also exist. When we explore error space by fixing $\lambda$ and fitting for the remaining parameters (Press et al. 2007), we find that specific combinations of parameters can replicate symmetric, flat-bottomed transits with spin-orbit misaligned planets. Those models contrive to have the planet traverse specific stellar chords that have nearly uniform flux due to combinations of gravity darkening and limb darkening, as shown in Figure 3 at bottom. While we cannot rule such fortuitous transit chords out, we consider them to be less probable than the spin-orbit aligned variants that show stronger robustness to the parameters that we held constant (i.e., $R_{*}, e$ ). Independent measurements of the stellar obliquity $\psi$, such as with asteroseismology, could resolve the degeneracy.

\section{DISCUSSION}

KOI-2138.01 represents just the second super-Earth candidate with a measured spin-orbit alignment and the first to be (probably) aligned. In Figure 4 we show the projected spinorbit alignment $\lambda$ for planets as a function of their radius. While we now have an appreciable understanding of the spin-orbit alignments of gas giants in short (less than 10 days) orbits, only a few planets smaller than Saturn have had their spin-orbit alignment determined. 


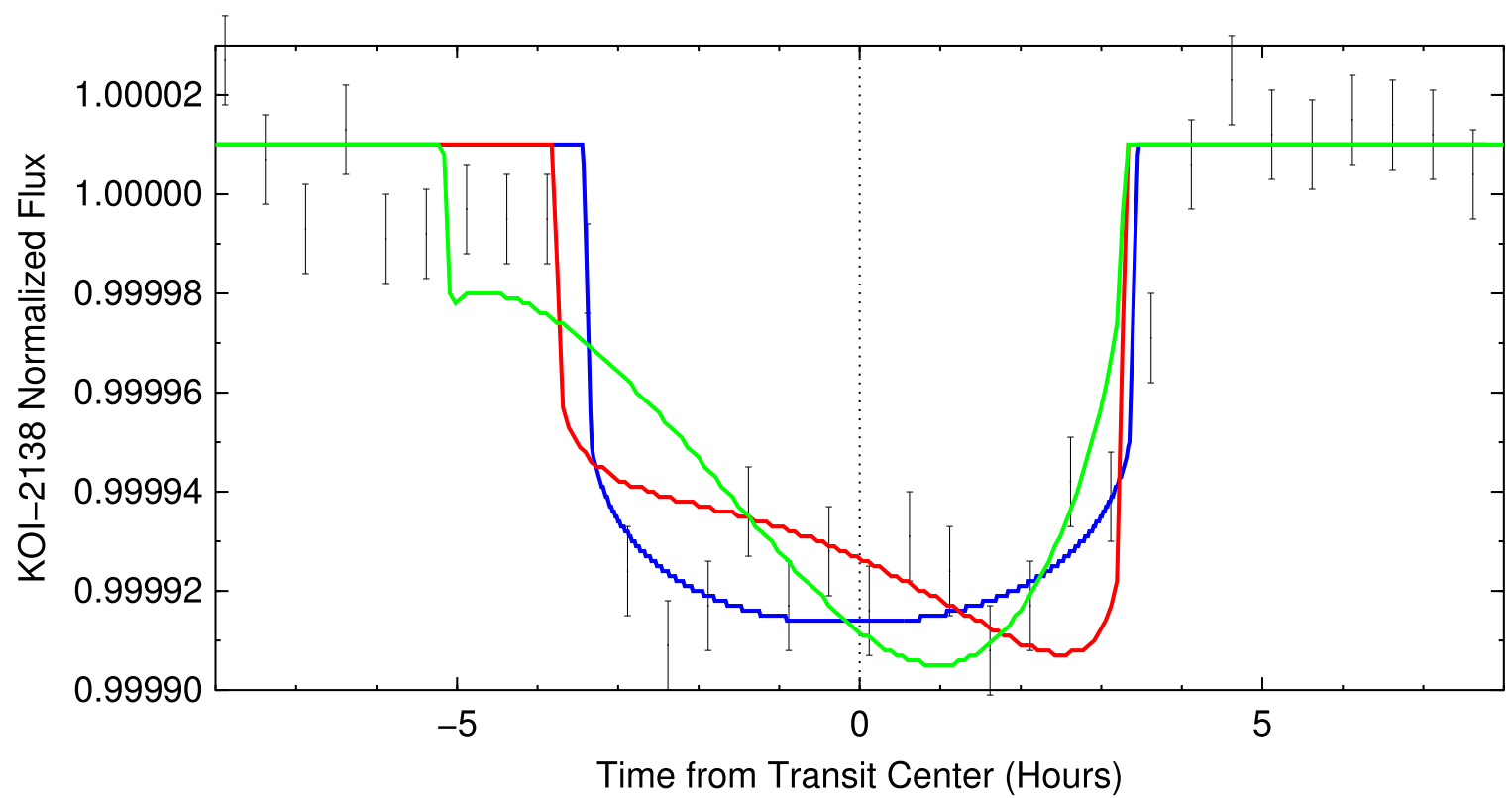

Figure 2. Here we show the Kepler lightcurve for KOI-2138, centered on the transit and folded with the planet candidate KOI-2138.01's 23.5541 day period. The data points in black are binned in time from the original to aid the in the evaluation of the fits. The lightcurve of our best-fit gravity-darkened model is shown in blue, along with two significantly misaligned models fit to the same data shown in red and green. The misaligned lightcurves serve to illustrate the capability of the model to discriminate between aligned and misaligned transits even with low signal-to-noise. The jaggedness in the lightcurves results from the limit of numerical precision of single-precision floating point numbers, as becomes important for very small transit depths such as that for KOI-2138.

Table 1

Best-fit Parameters for the KOI-2138 System

\begin{tabular}{lc}
\hline \hline Parameter & Best Fit Values \\
\hline$\chi_{\text {reduced }}^{2}$ & 1.0424 \\
$R_{*}$ & $2.286 R_{\odot}$ (fixed) \\
$M_{*}$ & $2.335 M_{\odot}$ (fixed) \\
$V \sin \psi$ & $200 \mathrm{~km} \mathrm{~s}^{-1}$ (fixed) \\
$\beta$ & 0.25 (fixed) \\
$R_{p}$ & $2.1 \pm 0.4 R_{\oplus}($ fit) \\
$i$ & $88^{\circ} .34 \pm 0.11$ (fit) \\
$c_{1}$ & 0.49 (fixed) \\
$c_{2}$ & 0 (fixed) \\
$e$ & 0 (fixed) \\
$\lambda$ & $1^{\circ} \pm 13^{\circ}$ (fit) \\
$\psi$ & $-4^{\circ} \pm 60^{\circ}$ (fit) \\
$P_{\text {rot }}$ & $14 \mathrm{hr}$ (derived) \\
$f_{*}$ & 0.10 (derived) \\
\hline
\end{tabular}

Note. The indicated value for the projected alignment $\lambda$ represents the formal $1-\sigma$ uncertainty from the covariance matrix - a more complete analysis allows for $\lambda=1_{-20}^{\circ}$, albeit with improbably lucky transit geometries (see the text).

The only other $\lambda$-measured super-Earth, $55 \mathrm{Cnc}$ e, has a 0.74 day misaligned orbit. Therefore if it is terrestrial, consistent with its Earth-like density, then 55Cnc e must be very different from any solar system planet. Certainly its close orbit drives a very high equilibrium temperature of over $1600 \mathrm{~K}$ (von Braun et al. 2011). Furthermore, that orbit is in fact so close-in that $55 \mathrm{Cnc}$ e almost certainly did not form in situ, and indeed its spin-orbit misalignment implies an interesting dynamical history as well.

Constrastingly, KOI-2138.01's longer-period (23.55 days), aligned orbit indicates that it could potentially be the first

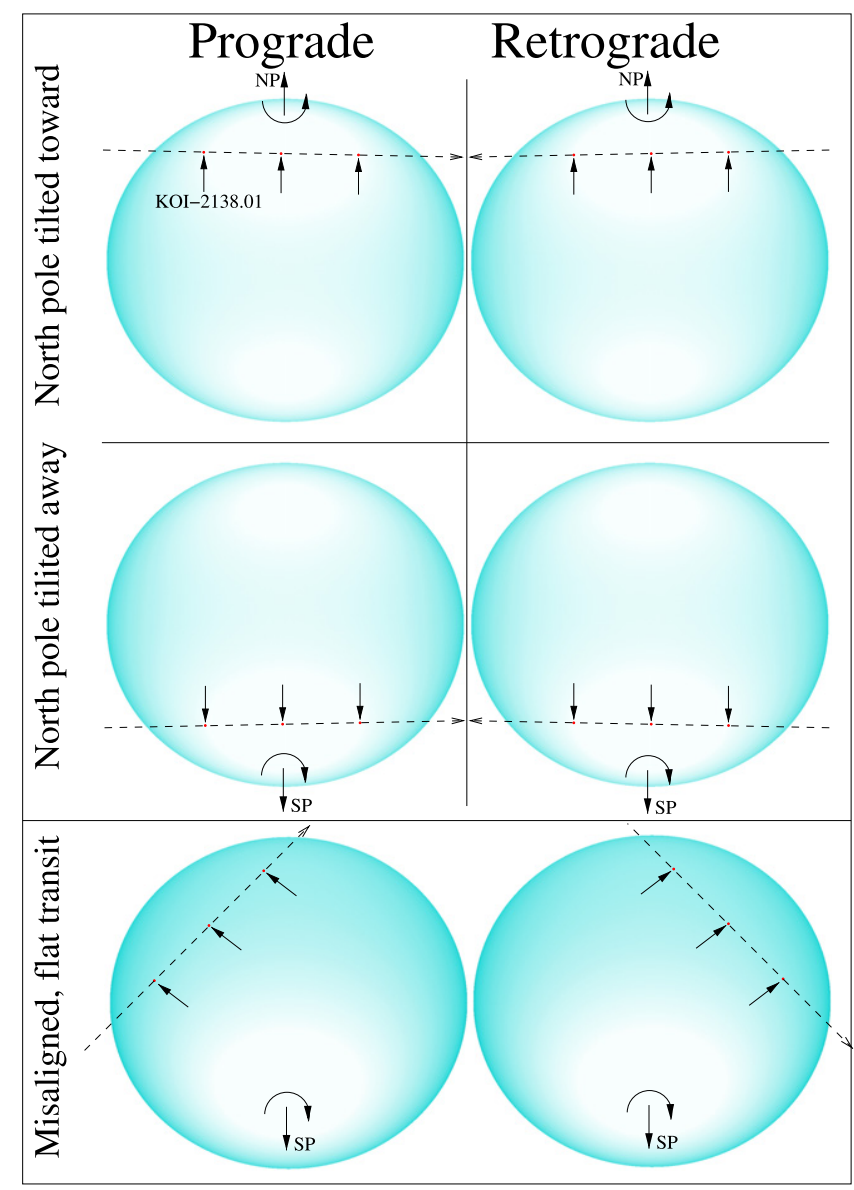

Figure 3. Our allowed transit geometries for KOI-2138.01. With small superEarth candidate KOI-2138.01 orbiting a relatively large, early-type star, the planet as shown here is tiny. We denote the planet's position with arrows and show it in red to make it more evident. 


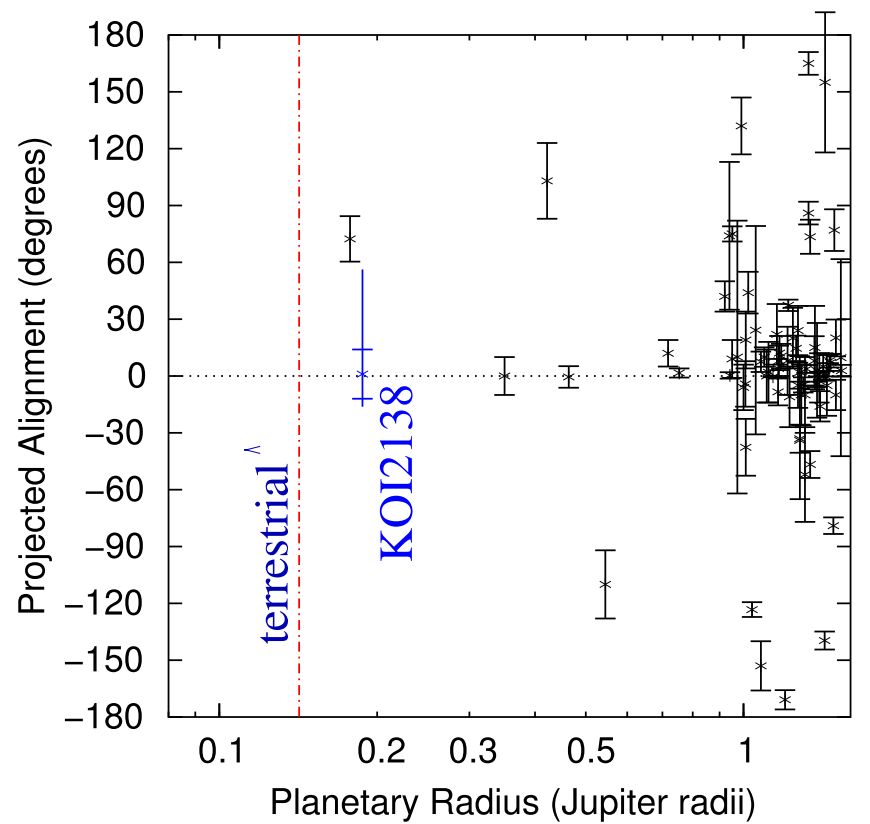

Figure 4. Here we plot the measured projected spin-orbit alignment $\lambda$ as a function of planetary radius $R_{p}$ in Jupiter radii $R_{\mathrm{Jup}}$. Each exoplanet with a useful measured constraint is shown on this plot, with data from René Heller's Holt-Rossiter-McLaughlin Encyclopaedia (www.physics.mcmaster.ca/ $\sim$ rheller). The red vertical line indicates the $1.6 R_{\oplus}$ cutoff above which all planets are volatile-rich according to Rogers (2014). KOI-2138, from this work, is indicated in blue and is the first spin-orbit aligned super-Earth candidate.

representative of an expected population of solar-system-like terrestrial planets. Its semimajor axis of $0.21 \mathrm{AU}$ puts KOI2138.01 inward of the Sun's Mercury by a factor of two, and with its hotter star KOI-2138.01 should still be hot with a subsolar $T_{\text {eff }}$ of $\sim 1300 \mathrm{~K}$. Terrestrial planet formation at such distances may be difficult, but given our lack of knowledge of planet formation around early-type stars this problem may not be insurmountable. If KOI-2138.01 did indeed form near its present location, then its aligned orbit may portend of a large population of rocky planets that we may be able to characterize in the coming decades.

The authors thank the anonymous reviewer for constructive comments. The authors acknowledge support from the NASA ADAP Program, grant \#NNX14AI67G. This study has made use of René Heller's Holt-Rossiter-McLaughlin Encyclopaedia (www.physics.mcmaster.ca/ rheller).

\section{REFERENCES}

Ahlers, J. P., Seubert, S. A., \& Barnes, J. W. 2014, ApJ, 786, 131 Albrecht, S., Winn, J. N., Johnson, J. A., et al. 2012, ApJ, 757, 18 Albrecht, S., Winn, J. N., Marcy, G. W., et al. 2013, ApJ, 771, 11 Barnes, J. W. 2009, ApJ, 705, 683

Barnes, J. W., Linscott, E., \& Shporer, A. 2011, ApJS, 197, 10

Barnes, J. W., van Eyken, J. C., Jackson, B. K., Ciardi, D. R., \& Fortney, J. J. 2013, ApJ, 774, 53

Batalha, N. M., Rowe, J. F., Bryson, S. T., et al. 2013, ApJS, 204, 24

Bieryla, A., Collins, K., Beatty, T. G., et al. 2015, AJ, in press (arXiv:1501.05565)

Bourrier, V., \& Hébrard, G. 2014, A\&A, 569, A65

Chaplin, W. J., Sanchis-Ojeda, R., Campante, T. L., et al. 2013, ApJ, 766, 101

Ciardi, D. R., van Belle, G. T., Akeson, R. L., et al. 2001, ApJ, 559, 1147

Collier Cameron, A., Bruce, V. A., Miller, G. R. M., Triaud, A. H. M. J., \& Queloz, D. 2010a, MNRAS, 403, 151

Collier Cameron, A., Guenther, E., Smalley, B., et al. 2010b, MNRAS, 407, 507

Crida, A., \& Batygin, K. 2014, A\&A, 567, A42

Dawson, R. I., \& Fabrycky, D. C. 2010, ApJ, 722, 937

Désert, J.-M., Charbonneau, D., Demory, B.-O., et al. 2011, ApJS, 197, 14

Djurašević, G., Rovithis-Livaniou, H., Rovithis, P., et al. 2003, A\&A, 402, 667

Fischer, D. A., Marcy, G. W., Butler, R. P., et al. 2008, ApJ, 675, 790

Gandolfi, D., Collier Cameron, A., Endl, M., et al. 2012, A\&A, 543, L5

Hirano, T., Narita, N., Sato, B., et al. 2012a, ApJL, 759, L36

Hirano, T., Sanchis-Ojeda, R., Takeda, Y., et al. 2012b, ApJ, 756, 66

Huber, D., Carter, J. A., Barbieri, M., et al. 2013, Sci, 342, 331

Johnson, J. A., Winn, J. N., Bakos, G. Á, et al. 2011, ApJ, 735, 24

Johnson, M. C., Cochran, W. D., Albrecht, S., et al. 2014, ApJ, 790, 30

Lissauer, J. J. 1993, ARA\&A, 31, 129

Lissauer, J. J., Fabrycky, D. C., Ford, E. B., et al. 2011, Natur, 470, 53

Masuda, K. 2015, ApJ, 805, 28

McArthur, B. E., Endl, M., Cochran, W. D., et al. 2004, ApJL, 614, L81

McLaughlin, D. B. 1924, ApJ, 60, 22

Miller, G. R. M., Collier Cameron, A., Simpson, E. K., et al. 2010, A\&A, 523, A52+

Monnier, J. D., Zhao, M., Pedretti, E., et al. 2007, Sci, 317, 342

Morton, T. D., \& Winn, J. N. 2014, ApJ, 796, 47

Nutzman, P. A., Fabrycky, D. C., \& Fortney, J. J. 2011, ApJL, 740, L10

Peterson, D. M., Hummel, C. A., Pauls, T. A., et al. 2006, Natur, 440, 896

Press, W. H., Teukolsky, S. A., Vetterling, W. T., \& Flannery, B. P. 2007, Numerical Recipes. The Art of Scientific Computing (3rd ed.; Cambridge: Cambridge Univ. Press)

Rogers, L. A. 2014, ApJ, in press (arXiv:1407.4457)

Rossiter, R. A. 1924, ApJ, 60, 15

Rowe, J. F., Coughlin, J. L., Antoci, V., et al. 2015, ApJS, in press (arXiv:1501.07286)

Sanchis-Ojeda, R., \& Winn, J. N. 2011, ApJ, 743, 61

Sanchis-Ojeda, R., Winn, J. N., Holman, M. J., et al. 2011, ApJ, 733, 127

Szabó, G. M., Szabó, R., Benkő, J. M., et al. 2011, ApJL, 736, L4+

Tregloan-Reed, J., Southworth, J., \& Tappert, C. 2013, MNRAS, 428, 3671

van Eyken, J. C., Ciardi, D. R., von Braun, K., et al. 2012, ApJ, 755, 42

von Braun, K., Boyajian, T. S., ten Brummelaar, T. A., et al. 2011, ApJ, 740,49

von Zeipel, H. 1924, MNRAS, 84, 665

Winn, J. N., Fabrycky, D., Albrecht, S., \& Johnson, J. A. 2010, ApJL, 718, L145

Zhou, G., \& Huang, C. X. 2013, ApJL, 776, L35 